\title{
Mechanism and kinetics of pore formation in membranes by water-soluble amphipathic peptides
}

\author{
Ming-Tao Lee ${ }^{\dagger}$, Wei-Chin Hung ${ }^{\ddagger}$, Fang-Yu Chen§, and Huey W. Huang ${ }^{\text {II| }}$ \\ ${ }^{\dagger}$ National Synchrotron Radiation Research Center, Hsinchu 30076, Taiwan; ${ }^{\ddagger}$ Department of Physics, Chinese Military Academy, Fengshan, Kaohsiung 83055, \\ Taiwan; §Department of Physics, National Central University, Chung-Li 32054, Taiwan; and "Department of Physics and Astronomy, Rice University, Houston, \\ TX 77251
}

Edited by Robert H. Austin, Princeton University, Princeton, NJ, and approved January 30, 2008 (received for review November 8, 2007)

\begin{abstract}
How antimicrobial peptides form pores in membranes is of interest as a fundamental membrane process. However, the underlying molecular mechanism, which has potential applications in therapeutics, nonviral gene transfer, and drug delivery, has been in dispute. We have resolved this mechanism by observing the time-dependent process of pore formation in individual giant unilamellar vesicles (GUVs) exposed to a melittin solution. An individual GUV first expanded its surface area at constant volume and then suddenly reversed to expanding its volume at constant area. The area expansion, the volume expansion, and the point of reversal all match the results of equilibrium measurements performed on peptide-lipid mixtures. The mechanism includes a negative feedback that makes peptide-induced pores stable with a well defined size, contrary to the suggestion that peptides disintegrate the membrane in a detergent-like manner.
\end{abstract}

antimicrobial peptides | membrane-thinning effect | stable membrane pore | peptide-induced pore | single-membrane experiment

$\mathbf{M}$ any water-soluble amphipathic peptides spontaneously bind to membranes and form transmembrane pores when the peptide concentrations exceed certain threshold values. Such pore-forming activities are of interest for many reasons. It is the common mode of action used by the ubiquitous antimicrobial peptides (1). A similar mechanism is used by pore-forming proteins, such as the apoptosis regulator Bcl-2-associated $\mathrm{X}$ protein (Bax), which activates pore formation in the outer mitochondria membrane to release the apoptotic factor cytochrome $c(2)$. Understanding the relatively simple process of pore formation by small peptides is an important step toward unraveling more complex membranous conformational changes such as membrane fusion (3). Clarifying the pore-forming mechanism will also facilitate its applications, including developing antimicrobial molecules as human therapeutics (1) or smallmolecule agents for nonviral gene transfer and drug delivery. The physical effects caused by the binding of pore-forming peptides to lipid bilayers have been studied by $\mathrm{x}$-ray and neutron diffraction on peptide-lipid mixtures (4-6). The results showed that peptide binding caused membrane thinning and pores appeared only when the thinning reached a critical fraction of the membrane thickness (4-7). However, the equivalent effects have not been demonstrated by kinetic experiments. This is important because pore formation in cell membranes caused by water-soluble peptides typically occurs as a kinetic process. Here, we report the observation of the time behavior of giant unilamellar vesicles (GUVs) exposed to the peptide melittin in solution. The observed time behavior of individual lipid vesicles, although complex, exhibits the physical effects seen in equilibrium experiments, thereby confirming that the mechanism of kinetic pore formation in single membranes is the same as that governing peptide-lipid interactions in the mixtures. It also implies that the same effect is likely to occur in cell membranes when the cells are exposed to these peptides. The GUV experiment clearly demonstrates that peptide-induced pores in membranes are stable with a well defined size.
Melittin, a peptide extracted from bee venom (8), and many antimicrobial peptides that have been studied (1) - e.g., magainin from the African clawed frog (9) and protegrin from pig leukocytes (10) - are similar in their antimicrobial activities and in their interactions with lipid vesicles $(6,11)$. The structural principle underlying all of these peptides is the ability of the molecule to adopt a conformation that segregates the hydrophobic and hydrophilic residues. Some of the peptides are naturally produced in amphipathic conformations stabilized by disulfide bonds-e.g., protegrin. Some adopt amphipathic conformations (typically $\alpha$-helices with amphipathic surfaces) only when bound to lipid bilayers-e.g., melittin and magainin. As a rule, the pore-forming peptides possess high binding affinities to the interface of lipid bilayers. However, binding and pore formation are separate events. The measured antimicrobial activities typically exhibit a sigmoidal concentration dependence (12): below a threshold concentration, there is little activity despite binding; but when the concentration exceeds the threshold, the activity rapidly reaches its maximum. The peptides' biological activities correlate with their abilities for causing content leakage from lipid vesicles (11). This and the fact that the D-enantiomers and the native L-peptides have identical activities (13), as well as other supporting evidence (12), strongly suggest that the target of antimicrobial peptides is the lipid matrix of cell membranes, rather than protein receptors. However, it is difficult to extract information for the molecular mechanism from activity experiments. The mechanism for pore formation remains controversial despite a large body of research. For example, a frequently cited proposition suggested that peptides disintegrate the membrane in a detergent-like action (14).

To gain insight into the action of peptides on a single membrane, we observed the time-dependent response of individual GUVs exposed to melittin solutions. We recorded and measured how each of $\approx 70$ GUVs responded to different concentrations of melittin, using the micropipette aspiration method (15). Two lipids of different chain lengths, 1,2-dierucoylsn-glycero-3-phosphocholine (di22:1PC) and 1,2-dieicosenoylsn-glycero-3-phosphocholine (di20:1PC), which produced stable GUVs, were chosen for this experiment. GUVs of each lipid produced a pattern of responses. To interpret the GUV results, we investigated the material properties of di20:1PC and di22:1PC bilayers containing melittin. From $x$-ray diffraction and oriented circular dichroism of the peptide-lipid mixtures, we measured the systematic change of bilayer properties as a function of melittin concentration. Such experiments with many other peptide-lipid combinations have been reported before $(5$,

Author contributions: M.-T.L., W.-C.H., F.-Y.C., and H.W.H. designed research; M.-T.L. and W.-C.H. performed research; M.-T.L., F.-Y.C., and H.W.H. analyzed data; and F.-Y.C. and H.W.H. wrote the paper.

The authors declare no conflict of interest.

This article is a PNAS Direct Submission.

\|To whom correspondence should be addressed. E-mail: hwhuang@rice.edu.

This article contains supporting information online at www.pnas.org/cgi/content/full/ 0710625105/DCSupplemental.

() 2008 by The National Academy of Sciences of the USA 

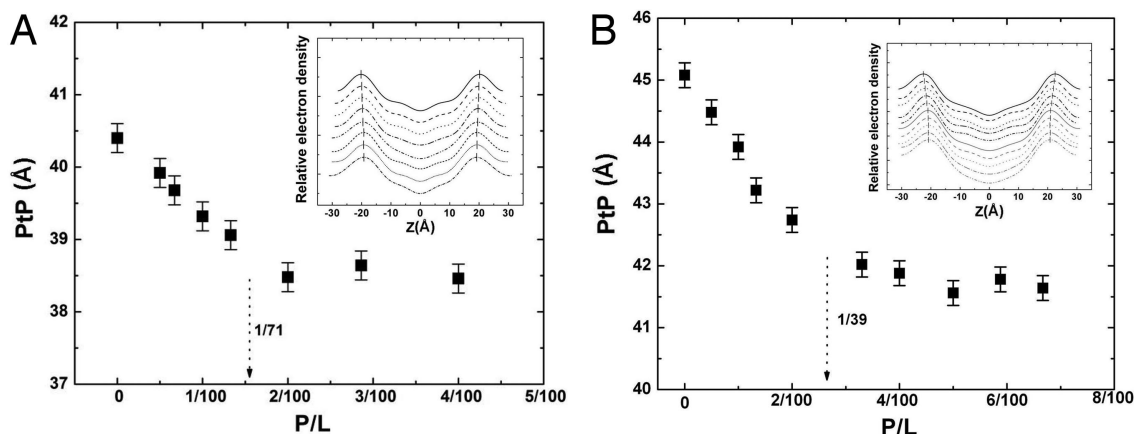

Fig. 1. Membrane-thinning effect by melittin. (A) di20:1PC bilayers containing melittin at the peptide-lipid molar ratios $\mathrm{P} / \mathrm{L}=0,1 / 200,1 / 150,1 / 100,1 / 75,1 / 50$, $1 / 35$, and $1 / 25$ measured by $x$-ray diffraction. $(B)$ di22:1PC bilayers containing melittin at $P / L=0,1 / 200,1 / 100,1 / 75,1 / 50,1 / 30,1 / 25,1 / 20,1 / 17$, and $1 / 15$ measured by $x$-ray diffraction [reproduced with permission from ref. 5 (Copyright 2004, American Chemical Society)]. (Insets) Electron-density profiles, from top to bottom (displaced for clarity) in the ascending order of P/L. The positions of the phosphate peaks are indicated. The peak-to-peak distance (PtP) is the measure of the bilayer thickness, shown as a function of P/L in the main panels. The error bars represent the variations by four independent measurements. The dotted arrows show the threshold concentrations for the peptide orientation change measured by OCD in Fig. 2, which approximately coincide with the onset positions of the thickness plateau in both cases.

7). We repeated the experiment here for these two lipids with melittin because the quantitative differences between different peptide-lipid combinations are important for a convincing interpretation of the GUV results.

\section{Results}

Membrane-Thinning Effect of Melittin. X-ray diffraction showed that the peptide-lipid mixtures formed homogeneous multiple bilayers. The electron-density profiles of the bilayers for a series of melittin and di20:1PC mixtures at different peptide-lipid molar ratios $\mathrm{P} / \mathrm{Ls}$ are shown in Fig. $1 A$ Inset. The profiles allow a precise measurement of the phosphate peak-to-phosphate peak distance $(P t P)$ as a measure of the bilayer thickness. Fig. $1 A$ shows that the bilayer thickness linearly decreases with $\mathrm{P} / \mathrm{L}$ and then levels off. Fig. $1 B$ shows the results for di22:1PC for comparison [from Lee et al. (5)].

Peptide Orientation Changes with Concentration. We also measured the circular dichroism of the peptides in the same samples used for x-ray experiment, with the multilayers oriented normal to the incident light (oriented circular dichroism) as shown in Fig. 2. Oriented circular dichroism (16) is sensitive to the peptide's helical orientation. It can be used to measure the fraction of helical peptides oriented parallel or perpendicular to the bilayers. We found that for melittin/di20:1PC mixtures, if the $\mathrm{P} / \mathrm{L}$ ratios are below a critical value $\mathrm{P} / \mathrm{L}^{*} \sim 1 / 70,100 \%$ of melittin helices were oriented parallel to the plane of bilayers, but for $\mathrm{P} / \mathrm{L}>\mathrm{P} / \mathrm{L}^{*}$ an increasing fraction of melittin helices became perpendicular to the bilayers (Fig. 2A). Very importantly, we found the value of $\mathrm{P} / \mathrm{L}^{*}$ coincidental with the onset of the bilayer thinning plateau (Fig. 1 $A$ ). Thus, both $\mathrm{x}$-ray and $\mathrm{CD}$ measurements found the same threshold $\mathrm{P} / \mathrm{L}^{*}$. This systematic behavior of peptide-lipid mixtures has been found for melittin in other lipid compositions, including di22:1PC (Fig. $2 B$ ), and also for other antimicrobial peptides including magainin and protegrin (5).

Multilamella of peptide-lipid mixtures were previously studied by neutron diffraction. Neutrons can detect the presence of water columns across the membrane, by $\mathrm{H}_{2} \mathrm{O}-\mathrm{D}_{2} \mathrm{O}$ exchange, if the membrane contained transmembrane pores. It was found repeatedly that the diffraction pattern included water columns of a well defined diameter ( $4.4 \mathrm{~nm}$ in the case of melittin) in the lipid bilayers when $\mathrm{P} / \mathrm{L}>\mathrm{P} / \mathrm{L}^{*}$, whereas no water columns were detected for $\mathrm{P} / \mathrm{L}<\mathrm{P} / \mathrm{L}^{*}(4,6)$. Clearly there are two distinct states of peptide in lipid bilayers as a function of $\mathrm{P} / \mathrm{L}$. At $\mathrm{P} / \mathrm{Ls}$ below $\mathrm{P} / \mathrm{L}^{*}$, melittin helices are all oriented parallel to the plane of bilayers-they do not form pores but cause membrane thinning in proportion to $\mathrm{P} / \mathrm{L}$. This implies that the melittin helices lie on the interface of the bilayer with their hydrophobic surfaces in contact with hydrocarbon chains while their polar surfaces face the lipid's polar headgroups. The inclusion of amphipathic peptides at the interface of the bilayer stretches the membrane area that forces the interior of the bilayer-i.e., the
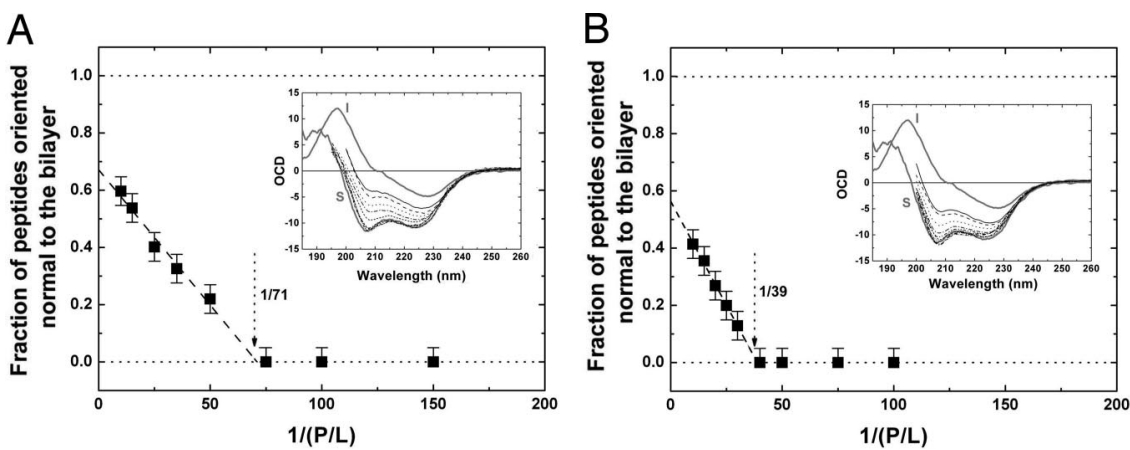

Fig. 2. Helical orientation of melittin in lipid bilayers. $(A$ and $B)$ Oriented circular dichroism (OCD) (Insets) of melittin embedded in di20:1PC bilayers at $P / L=$ $1 / 10,1 / 15,1 / 25,1 / 35,1 / 50,1 / 75,1 / 100$, and $1 / 150$ ( $A$; from top to bottom) and di22:1PC bilayers at $P / L=1 / 10,1 / 15,1 / 20,1 / 25,1 / 30,1 / 40,1 / 50,1 / 75$, and 1/100 [ $B$; reproduced with permission from ref. 5 (Copyright 2004, American Chemical Society)]. The spectra of melittin completely oriented normal (the I state) and parallel (the $S$ state) to the bilayers are shown in thick lines. Each intermediate spectrum was fit by a linear combination of $S$ and I to find the fraction of peptide oriented normal to the bilayers. (The error bars represent the standard deviations of the numerical fits.) These fractions are plotted as a function 1/(P/L) (main frames). In each graph, the linear fit to the values where the fractions are nonzero intercepts with the baseline to give the threshold $P / L *(5)$. 
hydrocarbon chain region-to become thinner but without breakage. Hydrocarbons have very small volume compressibility (17); therefore, the fractional area increase of a lipid bilayer is closely equal to its fractional thickness decrease. As $\mathrm{P} / \mathrm{L}$ increases above $\mathrm{P} / \mathrm{L}^{*}$, an increasing fraction of melittin helices become perpendicular to the bilayers, and pores are formed. In this state, the membrane thickness remains constant at its minimum value (Fig. 1), implying that the amount of peptide staying on the interface that has the effect of area-stretching remains constant at the threshold value $\mathrm{P} / \mathrm{L}^{*}$. All excessive peptides above $\mathrm{P} / \mathrm{L}^{*}$ are perpendicular to the bilayer and participate in pore formation.

Responses of GUVs to Melittin Binding. We now examine how the peptides interact with a GUV in a kinetic process. Single GUVs exposed to peptide solutions were observed under microscope using the micropipette aspiration method (15). We used the electroformation method (18) to produce GUVs in $100 \mathrm{mM}$ sucrose solution. An individual GUV was held at a low, constant tension by micropipette aspiration (15) and transferred to an isotonic glucose/sucrose solution containing a very low concentration of melittin. The process was recorded by video micrograph. Without melittin in the glucose/sucrose solution, the GUV remained unchanged. However, upon transfer to a solution with melittin, the GUV immediately underwent a surfacearea expansion, apparently due to its interaction with melittin molecules. [Such experiments were previously performed by Longo et al. (19), mainly with fusion peptides and briefly with melittin.] Fig. 3 shows the recorded images of GUVs in two different melittin concentrations. A phase condenser was used to record the phase contrast between the sucrose solution inside the GUV and the glucose/sucrose solution outside [see supporting information (SI) Methods]. A small amount of dye lipid on GUV allowed a precise measurement of the vesicle projection length $L_{\mathrm{P}}$ inside the micropipette and the radius of GUV (see Fig. S1).

We observed $\approx 70$ successful transfers of individual GUVs from the vesicle production chamber to the glucose/sucrose solution chamber containing various concentrations of melittin. The responses of GUVs are clearly different for melittin concentrations below or above $10^{-7} \mu \mathrm{M}$. In the majority of cases for melittin concentrations below $10^{-7} \mu \mathrm{M}$, the vesicle projection $L_{\mathrm{P}}$ increased with time to a saturated value, but the vesicle contrast remained unchanged (Fig. S2). This implied an increase of the vesicle surface area without a volume change, and the area change $\Delta A$ can be calculated from $\Delta L_{\mathrm{P}}: \Delta A=2 \pi R_{\mathrm{P}}(1-$ $\left.R_{\mathrm{P}} / R_{\mathrm{V}}\right) \Delta L_{\mathrm{P}}$, where $R_{\mathrm{P}}$ and $R_{\mathrm{V}}$ are, respectively, the radius of the micropipette and the radius of the $\operatorname{GUV}(15,19)$. To normalize the area changes for vesicles of different sizes, $\Delta L_{\mathrm{P}}$ was converted to the fractional change of vesicle area, $\Delta A / A$. The time-dependent $\Delta A / A$ for GUVs exposed to different concentrations of melittin is shown in Fig. 4. Although higher melittin concentrations tended to produce higher final $\Delta A / A$ values, the same melittin concentration could produce a range of different $\Delta A / A$. The final $\Delta A / A$ values for melittin concentrations below $10^{-7} \mu \mathrm{M}$ are mostly $<0.05$.

For melittin concentrations above $10^{-7} \mu \mathrm{M}$, there were two types of responses. The first type was similar to what was observed in low melittin concentrations except that the projection length $\Delta L_{\mathrm{P}}$ was longer and it took a longer time to reach a plateau value corresponding to final $\Delta A / A$ from 0.05 to 0.15 (Fig. 4). The second type initially had an increasing $\Delta L_{\mathrm{P}}$ just like the first type but then suddenly reversed to decreasing $L_{P}$ until the projection diminished. When $L_{\mathrm{P}}$ shortened, the contrast between the inside and outside of the vesicle also decreased (Fig. $\mathrm{S} 3$ ). We interpret this as being due to the presence of finite-sized pores in the membrane that allowed glucose, because it is smaller than sucrose, to permeate into the vesicle with cotransportation of water.
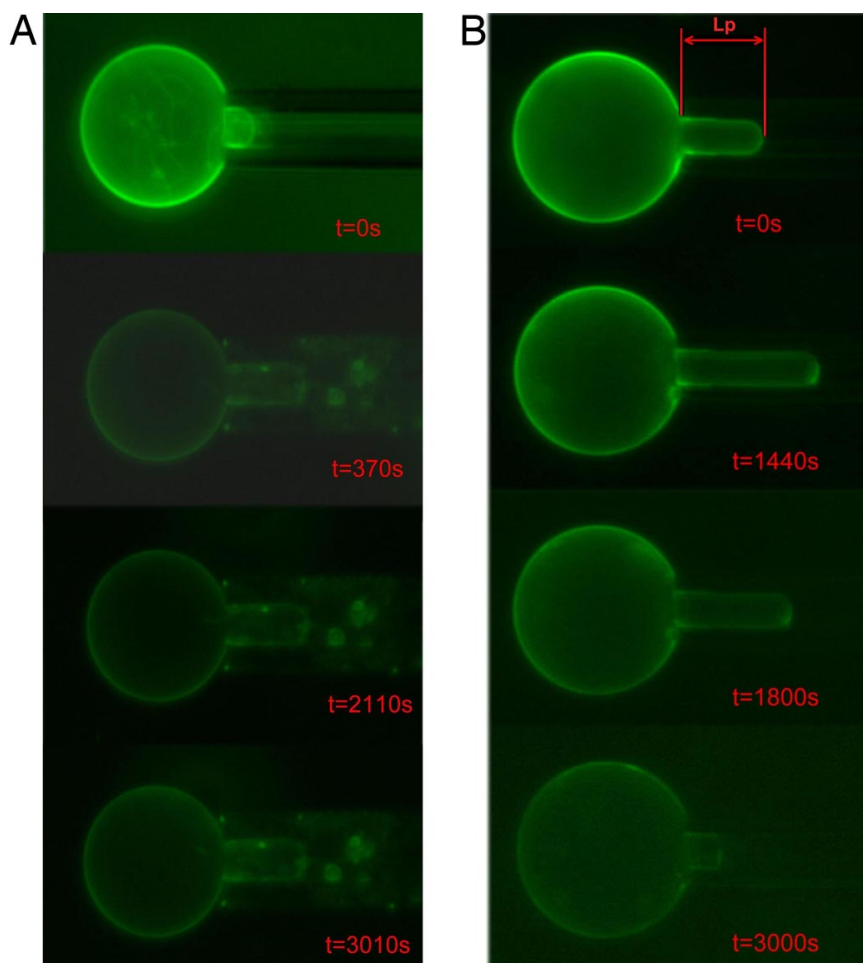

Fig. 3. Microscope images of GUV exposed to melittin. The typical GUV radius $R_{V}$ was $20-30 \mu \mathrm{m}$. The internal pipette radius $R_{\mathrm{P}}$ was 3-5 $\mu \mathrm{m}$. (A) At melittin concentration $2.15 \times 10^{-8} \mu \mathrm{M}$, the projection in the micropipette increased to a maximum length and remained constant in time. During the entire process, the contrast between the inside and outside of the vesicle remained unchanged (Fig. S2). (B) At melittin concentration $2.15 \times 10^{-6} \mu \mathrm{M}$, the projection first increased and then decreased until the projection diminished (the final image was faint because of photobleaching; data not shown) (also see Movie S1). The vesicle contrast relative to the solution was unchanged during the projection increase but decreased during the decrease of the projection length (Fig. S3).

\section{Discussion}

The kinetic experiment of GUVs can be understood from the results of equilibrium experiments. The area expansion of GUV was due to the adsorption of melittin molecules on the interface, the same effect detected as membrane thinning in equilibrium experiments. It is well known that melittin forms transient pores even at extremely low concentrations (20). Transient pores allow ion conduction driven by electric field but not water transport, and most importantly allow translocation of the peptide between two leaflets (11). Therefore, adsorbed melittin molecules always distributed to both sides of the lipid bilayer, allowing area expansion of both leaflets as observed. Initially, the vesicle projection continued to grow because more and more melittin molecules were adsorbed. If the ratio of the adsorbed peptide to lipid stayed below $\mathrm{P} / \mathrm{L}^{*}$, there was little chance of stable pore formation. In such cases, the vesicles reached a constant $\Delta A / A<$ 0.05 and remained intact for a long time, as in the majority of cases of GUVs exposed to melittin below $10^{-7} \mu \mathrm{M}$. The vesicle contrast did not change for the entire time (Fig. S2), and no stable pores were formed in the GUVs.

In equilibrium, when the peptide-lipid ratio exceeded the threshold value $\mathrm{P} / \mathrm{L}^{*}$, pores were always found in the membrane [by neutron diffraction $(4,6)$ ]. However, in a kinetic experiment, it is now well established (21-24), pore formation is probabilistic, depending on nucleation of defect in the lipid bilayer during the observation time. If there were no precursor defect in a GUV during the time of observation, no stable pores would form in the 

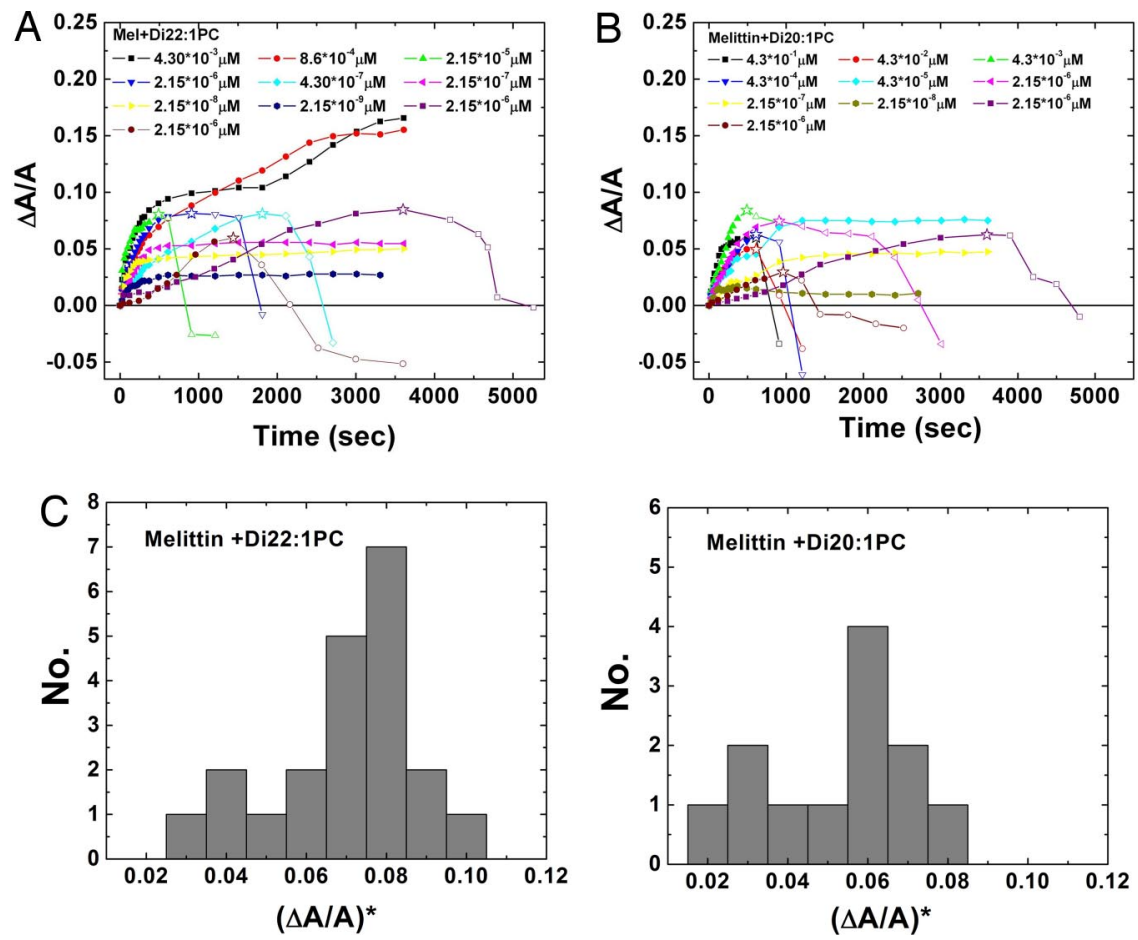

Fig. 4. Fractional area increase of GUV in time at various concentrations of melittin. Only a small number of representatives are shown here. The data for each run ended when the image became invisible because of photobleaching. ( $A$ ) GUVs of di22:1PC. The area increase $\Delta A$ was calculated from the increase of the projection length $\Delta L_{\mathrm{P}}$ at constant vesicle volume: $\Delta A=2 \pi R_{\mathrm{P}}\left(1-R_{\mathrm{P}} / R_{\mathrm{V}}\right) \Delta L_{\mathrm{P}}$. To keep the figure simple, the same relation was used to indicate a decrease in $\Delta L_{\mathrm{P}}$ by a decrease in $\Delta A$ [in fact, a negative $\Delta L_{p}$ is due to volume increase at constant area (see text)]. The beginning of projection decrease indicates the onset of pore formation (marked by a star). (B) Same as $A$ for lipid di20:1PC. (C) Histograms for the values of $\triangle A / A$ for the onset of pore formation, $(\Delta A / A) *$, from all measurements. The most frequently occurring $\triangle A / A$ values for the onset of pore formation are 0.08 and 0.06 in lipid di22:1PC and lipid di20:1PC, respectively.

GUV even if exposed to a high concentration of melittin. We have observed such cases where the area expansion of a GUV, because of a large amount of melittin adsorption, reached high values of $\Delta A / A$ from 0.05 to 0.15 . Again, the vesicle contrast remained unchanged in such cases. However, if nucleation of defect occurs in a GUV when the ratio of the adsorbed peptide to lipid exceeds $\mathrm{P} / \mathrm{L}^{*}$, the probability of pore formation is high. Unlike transient pores, the pores formed at a peptide concentration above $\mathrm{P} / \mathrm{L}^{*}$ are stable. They also have a well defined radius $(4,6)$ that allows permeation of small molecules like glucose more than for large molecules like sucrose. The volume of the vesicle increased in the presence of pores because of the influx of glucose with cotransportation of water due to osmosis, as evidenced by the decrease in the vesicle contrast (Fig. S3). We know from the equilibrium experiment that the membrane thickness remains constant once the pores are formed. Thus, the GUV with pores maintained a constant membrane area. The decrease in the projection length $\Delta L_{\mathrm{P}}$ was due to the volume increase at constant area: $\Delta V=-\pi R_{\mathrm{P}}\left(R_{\mathrm{V}}-R_{\mathrm{P}}\right) \Delta L_{\mathrm{P}}$. The onset of pore formation is marked by the point the vesicle projection began to decrease.

It is instructive to compare our results with tension-induced pore formation in GUVs without peptide. Experiments showed that pores do not form in a tensionless vesicle, but in a tense vesicle a pore may spontaneously appear (21-25). Such a pore is short-lived: it either closes or expands and ruptures the vesicle in a time scale of milliseconds or less (21-25). From these experiments, we came to understand that pore formation in a GUV is a kinetic process initiated by nucleation of precursor defect, rather than by thermal activation (21-25). When the tension was applied to a GUV by the micropipette aspiration method, the projection in the pipette increased with time until rupture (22). No decrease of the projection length was observed in this experiment. The rupture tension was found to distribute over a range of values depending on the loading rate of tension. Similarly in our GUV experiment, the value of $\Delta A / A$ for the onset of pore formation (where $L_{\mathrm{P}}$ began to decrease) distributes over a range (Fig. $4 C$ ). The most frequent pore-forming $\Delta A / A$ in di22:1PC and di20:1PC bilayers are, respectively, 0.08 and 0.06 (Fig. $4 C$ ). These values are consistent with the results of tensioninduced rupture experiment (22) where the most frequent rupture tension $\tau$ is related to most frequent rupture $\Delta A / A$, by the relation $\tau=K_{\mathrm{A}} \Delta A / A\left[K_{\mathrm{A}} \approx 240 \mathrm{mN} / \mathrm{m}\right.$ is the stretch modulus of lipid bilayers (26)], and the rupture $\Delta A / A$ systematically increased with membrane thickness (22).

The general agreement between the values of pore-forming $\Delta A / A$ and the rupture $\Delta A / A$ supports a previous conjecture that the mechanism for peptide-induced pore formation is closely related to tension-induced rupture of membrane (7). Both peptide adsorption and external tension have the same effect of expanding the membrane area, and rupture must start with the formation of a pore. The energy of a pore in a pure lipid vesicle is $E_{\mathrm{R}}^{0}=2 \pi r \gamma-\pi r^{2} \tau$ ( $r$ is the radius of the pore and $\gamma$ the line tension for the edge of the pore). This energy as a function of $r$ has a maximum, which is why a pore in a pure lipid membrane is unstable: for $r<\gamma / \tau$, the pore closes; for $r>\gamma / \tau$, the pore expands indefinitely until rupture, all within milliseconds or so in time (21-25). On the contrary, peptide-induced pores are stable. This is because the peptide-induced tension $\tau$ is due to the area expansion $\left(\tau=K_{\mathrm{A}} \Delta A / A\right)$ caused by peptide adsorption on the interface. When $\tau$ is large enough to induce pore formation, the pore formation in turn removes some peptide molecules from the interface to the edges of the pores, because of the high affinity for amphipathic molecules to bind to the edge [Gibbs's adsorption (25)] - this process relaxes the tension $\tau$ and stabilizes the pores. The action of peptides includes a mechanism of negative feedback for pore stabilization. As a result, peptide- 
induced pores have a well defined radius. (The mathematical proof is given in ref. 7.) Thus, external tension and amphipathic peptides both initiate pore formation by stretching the membrane, but the consequences of pore formation are different. A tension-induced pore expands and ruptures a GUV in milliseconds. Peptide-induced pores do not expand. As shown in the experiment, a slow glucose influx allowed GUVs with pores to survive for $>1.5 \mathrm{~h}$ (see Movie S1). This is contrary to the suggestion that peptides disintegrate membranes (14).

In the past, typical kinetic experiments were performed with a suspension of vesicles; for example, measuring the total content leakage from the vesicles when exposed to a peptide solution $(11,12,27)$. Such results are equivalent to taking the average of all of the GUV responses (some examples are shown in Fig. $4 A$ and $B$ ) and hence include the effect of poorly understood physics of defect (21-24). They did not reveal the molecular mechanism of pore formation. However, when we observed the responses of GUVs individually, we found that the pore formation in GUVs exhibits the same effects observed in equilibrium experiments. ( $i$ ) Binding of amphipathic peptide to the membrane interface stretches the membrane area. (ii) When the membrane is stretched beyond a threshold value of fractional area expansion, pores are formed. The most frequent pore-forming $\Delta A / A$ values 0.08 and 0.06 in kinetic experiments correspond to the equilibrium threshold $\Delta A / A=$ $0.094 \pm 0.006$ and $0.062 \pm 0.007$ measured by membrane thinning and OCD in di22:1PC and di20:1PC, respectively. (iii) Peptide-induced pores are stable with a well defined size. None of these could be seen from the average results obtained from a vesicle suspension.

In conclusion, individual GUV experiments reveal how melittin interacts with lipid bilayers and forms pores in a single membrane. The mechanism is predictable from the peptidelipid interactions observed from the studies of peptide-lipid mixtures. This mechanism explains the mode of action operated by antimicrobial peptides, the ubiquitous innate immune factors (1), and probably also by pore-forming proteins (2). We suggest that this knowledge can be used to design small molecular agents for therapeutics and for nonviral gene transfer or drug delivery. The method of combining the material study of mixtures and individual GUV experiment is a promising method for the study of membrane-active molecules, including drugs and proteins.

\section{Methods}

Materials. 1,2-Dierucoyl-sn-glycero-3-phosphocholine (di22:1PC), 1,2dieicosenoyl-sn-glycero-3-phosphocholine (di20:1PC), and 1,2-dioleoyl-snglycero-3-phosphoethanolamine- $N$-(7-nitro-2-1,3-benzoxadiazol-4-yl) (18:1 NBD PE) were purchased from Avanti Polar Lipids. Melittin was purchased from Sigma-Aldrich. In previous experiments on melittin-lipid interactions performed by Yang et al. (6), no difference was found between Sigma melittin and pure synthetic melittin.

X-Ray Diffraction. We prepared homogeneous mixtures of lipid and melittin in the form of oriented multilayers, a stack of parallel lipid bilayers on a solid substrate. The preparation of such oriented samples followed the method described in previous studies (28). The sample was then placed in a temperature-humidity chamber, in which the water vapor pressure was controlled by the concentration of polyethylene glycol in a water bath (29). The measurement was made at $30^{\circ} \mathrm{C}, 98 \%$ relative humidity. [At higher relative humidity, the quality of diffraction pattern deteriorates because of undulation fluctuations of the membranes (30).] Diffraction was measured with a laboratory $\mathrm{x}$-ray diffractometer (31). Only samples that produced at least five discernible diffraction peaks were accepted (Fig. S4). Each peptide-lipid combination was measured with at least two separately prepared samples. Each sample was measured twice separately to check the reproducibility. The procedure for data reduction was described in many of our previous papers, including the phase determination $(28,30)$ (Fig. S5). The diffraction amplitudes were then converted to the electron-density profiles across the bilayer (Insets of Fig. $1 \mathrm{~A}$ and $B$ ). The primary purpose of $\mathrm{x}$-ray diffraction is to measure the phosphate peak-to-phosphate peak distance across the bilayer $(P t P)$ as a measure of the bilayer thickness. The diffraction patterns and the electron-density profiles also verified that the peptide-lipid mixtures formed homogeneous bilayers.

The thickness of the hydrocarbon region $h$ is PtP $-10 \AA$, or PtP minus twice the length of the glycerol region (from the phosphate to the first methylene of the hydrocarbon chains) (5). Because the volume compressibilities of lipids are very small (32), the fractional decrease of the hydrocarbon thickness is closely equal to the fractional area increase $-\Delta h / h=\Delta A / A$. From the membrane thinning data (Fig. 1), we found the threshold $\triangle A / A$ at $P / L^{*}$ for melittin in di20:1PC, $-\Delta h / h=\Delta A / A=0.062 \pm 0.007$. For melittin in di22:1PC, we found at the threshold $\mathrm{P} / \mathrm{L}^{*},-\Delta h / h=\Delta A / A=0.094 \pm 0.006$.

Oriented Circular Dichroism (OCD). The principle of orientation-dependent CD and the method of OCD measurement were introduced in ref. 16. The same samples prepared for $x$-ray diffraction were put in a CD spectropolarimeter (Jasco J-810) and measured with the incident light normal to the substrate. The background OCD spectra of pure lipid bilayers were measured separately and were removed from the spectra of the corresponding samples containing peptides.

The OCD spectra for melittin helices completely parallel (the S state) or completely perpendicular (the I state) to the plane of bilayers were measured in DMPC bilayers (29). Normalization between spectra is described in details in previous papers $(29,33)$. The OCD of melittin in di20:1PC and di22:1PC were limited to above $\approx 200 \mathrm{~nm}$ (whereas in DMPC the spectra extend to below 180 $\mathrm{nm}$ ) because unsaturated hydrocarbons have stronger UV absorbance. Each melittin OCD was fit to a linear combination of the S spectrum and the I spectrum to estimate the fraction of melittin helices in the $\mathrm{S}$ or the I state.

GUVs Experiment. GUVs were produced in $100 \mathrm{mM}$ sucrose solution by the electroformation method (18). Then an isotonic glucose solution was added to the production chamber so that the molar ratio of glucose to sucrose was 3:4 outside the vesicles. (This particular mixture was chosen so that when pores are formed in GUVs, the influx of glucose into the GUVs was slow enough that the GUVs could be observed for at least $1.5 \mathrm{~h}$.) The same glucose/sucrose mixture filled a separate observation chamber with various concentrations of melittin. For each experiment, one GUV was aspirated at a constant tension (0.1-0.5 $\mathrm{dyn} / \mathrm{cm}$ ) and held steady. Using the microscope stage, the chambers were translated so that the GUV was transferred into the observation chamber (see SI Methods).

The purpose of our experiment was to observe the responses of GUVs as a function of $\triangle A / A$, not as a function of melittin concentration in the solution. Note that the melittin concentrations used in this experiment $\left(10^{-9}\right.$ to $10^{-3}$ $\mu \mathrm{M})$ were much lower than those used in bioactivity assays or leakage experiments, which are typically in the micromolar range. In a leakage experiment, the peptide-to-lipid molar ratios of the entire suspension were typically in the range of $0.005-0.1$ (11). A similar range of peptide-to-lipid molar ratios was used in our experiment. For example, if the GUV radius was $25 \mu \mathrm{m}$ and the melittin concentration was $10^{-6} \mu \mathrm{M}$ (total volume $560 \mu \mathrm{l}$ ), the peptide-to-lipid molar ratio was $\approx 1 / 50$. When melittin concentrations in the micromolar range were used, we found in most cases that GUVs ruptured instantly as soon as they were transferred to the observation chamber.

A change in the surface area of a GUV in terms of $\Delta L_{P}$ and $\Delta R_{V}$ is given by $\Delta A=2 \pi R_{\mathrm{P}} \Delta L_{\mathrm{P}}+8 \pi R_{\mathrm{V}} \Delta R_{\mathrm{V}}$. A change in the GUV volume is given by $\Delta V=$ $\pi R_{\mathrm{P}}^{2} \Delta L_{\mathrm{P}}+4 \pi R_{\mathrm{V}}^{2} \Delta R_{\mathrm{V}}$. Therefore, if the surface area is changing at constant volume, one has $\Delta A=2 \pi R_{\mathrm{P}}\left(1-R_{\mathrm{P}} / R_{\mathrm{V}}\right) \Delta L_{\mathrm{P}}$, whereas if the volume is changing at constant surface area, one has $\Delta V=-\pi R_{\mathrm{P}}\left(R_{\mathrm{V}}-R_{\mathrm{P}}\right) \Delta L_{\mathrm{P}}$.

The sucrose solution and the glucose solution have different indices of refraction. The phase contrast between the inside and outside of the vesicle was used to monitor the possible change of the vesicle content. A phase condenser was used to record the phase-contrast images. However, the projection in the micropipette could not be seen clearly by the phase-contrast image. We had to observe the change of GUV projection by fluorescence images. So we measured the phase contrast for every run at the beginning and at the end. For a number of runs, we interrupted the fluorescence image recording and measured the phase contrast in the middle of the run. We found that the vesicle contrast did not change as long as the projection had not decreased. However, if the GUV projection length decreased, the vesicle contrast always decreased (see examples in Figs. S2 and S3).

ACKNOWLEDGMENTS. We thank Ping Chang for performing part of the GUV experiments. This work was supported by Taiwan National Science Council Contracts NSC 96-2112-M-213-007-MY3 (to M.-T.L.), NSC 95-2112-M-145-001 (to W.-C.H.), and NSC 95-2112-M-008-018-MY3 (to F.-Y.C.); National Institutes of Health Grant GM55203 (to H.W.H.); and Robert A. Welch Foundation Grant C-0991 (to H.W.H.). 
1. Zasloff M (2002) Antimicrobial peptides of multicellular organisms. Nature 415:389395

2. Garcia-Saez AJ, Chiantia S, Salgado J, Schwille P (2007) Pore formation by a Bax-derived peptide: Effect on the line tension of the membrane probed by AFM. Biophys $J$ 93:103-112.

3. Martens S, MM. Kozlov, McMahon HT (2007) How synaptotagmin promotes membrane fusion. Science 316:1205-1208.

4. Ludtke SJ, et al. (1996) Membrane pores induced by magainin. Biochemistry 35:1372313728.

5. Lee M-T, Chen F-Y, Huang HW (2004) Energetics of pore formation induced by membrane active peptides. Biochemistry 43:3590-3599.

6. Yang L, Harroun TA, Weiss TM, Ding L, Huang HW (2001) Barrel-stave model or toroidal model? A case study on melittin pores. Biophys $J$ 81:1475-1485.

7. Huang HW, Chen F-Y, Lee M-T (2004) Molecular mechanism of peptide induced pores in membranes. Phys Rev Lett 92:198304.

8. Habermann E (1972) Bee and wasp venoms. Science 177:314-322.

9. Zasloff M (1987) Magainins, a class of antimicrobial peptides from Xenopus skin: Isolation, characterization of two active forms, and partial cDNA sequence of a precursor. Proc Natl Acad Sci USA 84:5449-5453.

10. Kokryakov VN, et al. (1993) Protegrins: Leukocyte antimicrobial peptides that combine features of corticostatic defensins and tachyplesins. FEBS Lett 327:231-236.

11. Matsuzaki K, Yoneyama S, Miyajima K (1997) Pore formation and translocation of melittin. Biophys J 73:831-838.

12. Boman HG, Marsh J, Goode JA, eds (1994) Antimicrobial Peptides (Wiley, Chichester, UK).

13. Wade $D$, et al. (1990) All-D amino acid-containing channel-forming antibiotic peptides. Proc Natl Acad Sci USA 87:4761-4765.

14. Shai $Y$ (1999) Mechanism of the binding, insertion and destabilization of phospholipid bilayer membranes by $\alpha$-helical antimicrobial and cell non-selective membrane-lytic peptides. Biochim Biophys Acta 1462:55-70.

15. Kwok R, Evans E (1981) Thermoelasticity of large lecithin bilayer vesicles. Biophys J 35:637-652

16. Wu Y, Huang HW, Olah GA (1990) Method of oriented circular dichroism. Biophys J 57:797-806.

17. Tosh RE, Collings PJ (1986) High pressure volumetric measurements in dipalmitoylphosphatidylcholine bilayers. Biochim Biophys Acta 859:10-14.
18. Angelova MI (2000) Liposome electroformation. Giant Vesicles, eds Luisi PL, Walde P (Wiley, Chichester, UK), pp 27-36.

19. Longo ML, Waring AJ, Gordon LM, Hammer DA (1998) Area expansion and permeation of phospholipid membrane bilayer by influenza fusion peptides and melittin. Lang muir 14:2385-2395.

20. Tosteson MT, Tosteson DC (1981) The sting. Melittin forms channels in lipid bilayers. Biophys J 36:109-116.

21. Brochard-Wyart F, de Gennes PG, Sander O (2000) Transient pores in stretched vesicles: Role of leak-out. Physica A 278:32-51.

22. Evans E, Heinrich V, Ludwig F, Rawicz W (2003) Dynamic tension spectroscopy and strength of biomembranes. Biophys J 85:2342-2350.

23. Karatekin EO, et al. (2003) Cascades of transient pores in giant vesicles: Line tension and transport. Biophys J 84:1734-1749.

24. Melikov KC, et al. (2001) Voltage-induced nonconductive pre-pores and metastable single pores in unmodified planar lipid bilayer. Biophys $J$ 80:1829-1836.

25. Puech PH, Borghi N, Karatekin E, Brochard-Wyart F (2003) Line thermodynamics: adsorption at a membrane edge. Phys Rev Lett 90:128304.

26. Rawicz W, Olbrich KC, McIntosh T, Needham D, Evans E (2000) Effect of chain length and unsaturation on elasticity of lipid bilayers. Biophys $J$ 79:328-339.

27. Andersson A, Danielsson J, Graslund A, Maler L (2007) Kinetic models for peptideinduced leakage from vesicles and cells. Eur Biophys J 36:621-635.

28. Ludtke S, He K, Huang H (1995) Membrane thinning caused by magainin 2. Biochemistry 34:16764-16769.

29. Chen F-Y, Lee M-T, Huang HW (2003) Evidence for membrane thinning effect as the mechanism for peptide-induced pore formation. Biophys J 84:3751-3758.

30. Chen F-Y, Hung W-C, Huang HW (1997) Critical swelling of phospholipid bilayers. Phys Rev Lett 79:4026-4029.

31. Weiss TM, van der Wel PC, Killian JA, Koeppe, RE, II, Huang HW (2003) Hydrophobic mismatch between helices and lipid bilayers. Biophys $J$ 84:379-385.

32. Seemann H, Winter R (2003) Volumetric properties, compressibilities, and volume fluctuations in phospholipid-cholesterol bilayers. Z Phys Chem 217:831-846.

33. Chen F-Y, Lee M-T, Huang HW (2002) Sigmoidal concentration dependence of antimicrobial peptide activities: a case study on alamethicin. Biophys J 82:908-914. 